\title{
Dimensional crossover of hard parallel cylinders confined on cylindrical surfaces
}

\author{
Yuri Martínez-Ratón* \\ Grupo Interdisciplinar de Sistemas Complejos (GISC), Departamento de Matemáticas, Escuela Politécnica Superior, \\ Universidad Carlos III de Madrid, Avenida de la Universidad 30, E-28911, Leganés, Madrid, Spain \\ Enrique Velasco ${ }^{\dagger}$ \\ Departamento de Física Teórica de la Materia Condensada and Instituto de Ciencia de Materiales Nicolás Cabrera, \\ Universidad Autónoma de Madrid, E-28049 Madrid, Spain
}

(Received 5 March 2013; published 31 May 2013)

\begin{abstract}
We derive, from the dimensional-crossover criterion, a fundamental-measure density functional for parallel hard curved rectangles moving on a cylindrical surface. We derive it from the density functional of circular arcs of length $\sigma$ with centers of mass located on an external circumference of radius $R_{0}$. The latter functional in turn is obtained from the corresponding two-dimensional functional for a fluid of hard disks of radius $R$ on a flat surface with centers of mass confined onto a circumference of radius $R_{0}$. Thus the curved length of closest approach between the two centers of mass of hard disks on this circumference is $\sigma=2 R_{0} \sin ^{-1}\left(R / R_{0}\right)$, the length of the circular arcs. From the density functional of circular arcs, and by applying a dimensional expansion procedure to the spatial dimension orthogonal to the plane of the circumference, we finally obtain the density functional of curved rectangles of edge lengths $\sigma$ and $L$. Along with the derivation, we show that, when the centers of mass of the disks are confined to the exterior circumference of a circle of radius $R_{0}$, (i) for $R_{0}>R$, the exact Percus one-dimensional (1D) density functional of circular arcs of length $2 R_{0} \sin ^{-1}\left(R / R_{0}\right)$ is obtained, and (ii) for $R_{0}<R$, the zero-dimensional limit (a cavity that can hold one particle at most) is recovered. We also show that, for $R_{0}>R$, the obtained functional is equivalent to that of parallel hard rectangles on a flat surface of the same lengths, except that now the density profile of curved rectangles is a periodic function of the azimuthal angle, $\rho(\phi, z)=\rho(\phi+2 \pi, z)$. The phase behavior of a fluid of aligned curved rectangles is obtained by calculating the free-energy branches of smectic, columnar, and crystalline phases for different values of the ratio $R_{0} / R$ in the range $1<R_{0} / R \leqslant 4$; the smectic phase turns out to be the most stable except for $R_{0} / R=4$, where the crystalline phase becomes reentrant in a small range of packing fractions. When $R_{0} / R<1$ the transition is absent, since the density functional of curved rectangles reduces to the 1D Percus functional.
\end{abstract}

DOI: 10.1103/PhysRevE.87.052314

\section{INTRODUCTION}

The subjects of surfaces decorated with particles in periodically or quasiperiodically packed configurations and the arrangement of spheres on spherical, cylindrical, or general surfaces have attracted a long-standing interest $[1,2]$. The focus has been mainly on the type of packing, defect stabilization and interactions, and the topological constraints associated with nonvanishing Gaussian curvature. A most prominent problem concerns the stabilization of crystalline order on a sphere, first predicted [3] and then experimentally observed in colloidal spheres on water droplets [4]. The problem has many important consequences in a number of fields, for example, possible packings of protein capsomeres in spherical viruses [5], the behavior of particles inserted in lipid membranes and their curvature-induced interactions [6,7], metallic clusters, the structure of fullerenes, to name just a few. The spherical or effectively spherical surface is the most studied, while several studies have also appeared on cylindrical surfaces. Recently, Nelson studied the interaction of dislocations on a cylindrical surface [8]; despite the vanishing Gaussian curvature of the cylinder, a rich phenomenology was found.

Of particular interest is the case when particles are not spherical but elongated, since here there are issues of packing not only from the translational but also from the orientational

\footnotetext{
*yuri@math.uc3m.es

†enrique.velasco@uam.es
}

PACS number(s): 61.20.Gy, 61.30.Cz, 47.57.J-

degrees of freedom. Hence one has here two fields, the position and the nematic-director fields, that compete and interact with the geometry to possibly stabilize complex defected patterns. In a nematic phase particle positions are disordered but the nematic director is still constrained by the topology. The possibility of stabilizing defects by confining a thin nematic film on a spherical surface is intriguing. For example, using computer simulations, Dzubiella et al. [9] studied the nematic ordering of hard rods confined to be on the surface of a sphere. In this case the confinement on a spherical surface induces a global topological charge in the director field due to the stabilization of half-integer topological point defects.

Another interesting, much less analyzed, aspect concerns the formation of phases with partial positional order (liquid crystalline) from a disordered phase and the conditions imposed by topology, curvature, and periodicity on the ordering and phase interplay as thermodynamic conditions (such as surface density of particles or temperature) are varied. In liquid-crystalline phases with partial or total translational order (smectic, columnar, or crystalline) the two fields are coupled, and a complex interaction with the topology may result. This problem may be relevant in connection with the ordering of large protein molecules inserted into lipid membranes, where curvature may both induce or modify order and be induced or modified by order.

In the present article we focus on a system of parallel circular, rectangular particles confined to a cylindrical surface 
(therefore the nematic phase is the most disordered phase of the system). This is motivated as a useful model to discuss ordering of squared or rectangular proteins or otherwise on rod-shaped bacterial cell membranes, but the model can be analyzed in a broader context. Despite its simple topology, the nonvanishing curvature and periodicity perpendicular to the cylinder axis may induce or suppress ordering in some of the two orthogonal directions, giving rise to possible smectic or columnar orderings of the particles on the cylindrical surface. This problem has some similarities with the adsorption of particles inside slitlike or cylindrical pores [an example of which is the recent study on the confinement of hard spheres (HSs) into cylindrical pores [10], or of hard rods into planar pores [11,12], both employing the density-functional (DF) formalism]. Recently the close-packed structures of HSs confined in cylindrical pores of small radii were classified using analytical methods and computer simulations [13]. All of these studies reflect the importance of the commensuration between the pore width (in our case the circle diameter) and the characteristic dimensions of particles in the structure and stability of the confined nonuniform phases. Recent studies show that the extreme confinement of particles along one direction makes the system behave as an ideal gas in this direction. Thus the corresponding degrees of freedom can be integrated out and the resulting lower-dimensional system can be described by an effective interparticle potential [14].

The purpose of the present article is twofold. First, we derive a density-functional theory for a fluid of parallel circular rectangles on an external cylindrical surface; this model is isomorphic to a fluid monolayer of parallel cylinders adsorbed on an external cylindrical surface, or to the same fluid confined between two concentric cylindrical surfaces such that only one shell of cylinders can be accommodated with no radial motion. We show that the functional may be obtained consistently from different routes due to the important dimensional-crossover property of the theory. This property was first used to derive a fundamental-measure density functional (FMF) for HSs $[15,16]$ and parallel hard cubes (PHCs) [17], and it was recently applied to obtain a density functional for hard parallel cylinders [18]. Second, the model is analyzed statistical-mechanically by investigating the free-energy minima landscape. We discuss different regimes for the ratio of the radius of the external cylinder to radii of the underlying adsorbed cylinders. When the ratio is sufficiently low the model is an effectively one-dimensional (1D) model and no phase transition exists. Otherwise the smectic phase is found to be the most stable except for a relatively small range of densities in which the crystalline phase appears as a reentrant phase. These results are in line with those of recent experiments on confined liquid crystals in silica-glass nanochannels, which show that the stability of the smectic phase is considerably enhanced by the confinement at the expense of the crystalline phase [19]. We finally propose, following the dimensional-crossover property, a DF for spherical lenses (the intersection between a HS of radius $R$ whose center of mass is located on an external sphere of radius $R_{0}$, with $R_{0}>R$ ) moving on a spherical surface.

The article is arranged as follows. In Sec. II we derive the density-functional theory for hard curved rectangles (CRs) on an external cylindrical surface. This is done in two steps: In the first (Sec. II A), the two-dimensional (2D) functional for hard disks (HDs) is projected on the surface of an external circle, thus obtaining a 1D functional for curved arcs (CAs) that move along the circumference of the external circle. In the second (Sec. II B) the functional is developed along the direction perpendicular to the circle to give a 2D functional for CRs. Dimensional consistency is discussed in Sec. II C. The results are presented in Sec. III. In Sec. V we propose, following the same dimensional-crossover recipe, a DF for spherical lenses whose centers of mass are confined on an external sphere. Finally some conclusions are presented in Sec. VI. Details on the derivation of the density functionals and the density profile parametrizations are relegated to Appendixes A-C.

\section{DERIVATION OF FUNCTIONALS}

In this section we derive the FMF for our model system, i.e., a fluid of CRs. We do it in two steps. First, the 2D functional for an HD of radius $R$ is projected on the external surface of a cylinder of radius $R_{0}$, providing a $1 \mathrm{D}$ functional for a fluid of CAs (Sec. II A). Then, this 1D functional is developed along the orthogonal dimension to obtain a $2 \mathrm{D}$ functional for the CR fluid (Sec. II B). The two cases $R_{0}<R$ and $R_{0}>R$ are discussed separately in each case, since they give rise to fundamentally different expressions. In the last part of this section the resulting functionals are shown to be dimensionally consistent.

\section{A. Functional for CA}

We start from the FMF excess free-energy for a fluid of HDs derived in Refs. [16,18], i.e.,

$$
\beta \mathcal{F}_{\mathrm{ex}}^{(\mathrm{HD})}[\rho]=\int d \mathbf{r} \Phi_{2 \mathrm{D}}^{(\mathrm{HD})}(\mathbf{r}),
$$

where $\mathbf{r}=(r, \phi)$ is the radius vector in polar coordinates. The reduced free-energy density is defined as

$$
\Phi_{2 \mathrm{D}}^{(\mathrm{HD})}(\mathbf{r})=-n_{0}^{(\mathrm{HD})}(\mathbf{r}) \ln \left[1-\eta_{\mathrm{HD}}(\mathbf{r})\right]+\frac{N_{\mathrm{HD}}(\mathbf{r})}{1-\eta_{\mathrm{HD}}(\mathbf{r})},
$$

where the weighted densities are convolutions of the twodimensional density profile $\rho_{2 \mathrm{D}}(\mathbf{r})$ :

$$
\begin{gathered}
n_{0}^{(\mathrm{HD})}(\mathbf{r})=\frac{1}{2 \pi R} \int d \mathbf{r}_{1} \rho_{2 \mathrm{D}}\left(\mathbf{r}_{1}\right) \delta\left(R-\left|\mathbf{r}-\mathbf{r}_{1}\right|\right), \\
\eta_{\mathrm{HD}}(\mathbf{r})=\int d \mathbf{r}_{1} \rho_{2 \mathrm{D}}\left(\mathbf{r}_{1}\right) \Theta\left(R-\left|\mathbf{r}-\mathbf{r}_{1}\right|\right), \\
N_{\mathrm{HD}}(\mathbf{r})=\frac{1}{(2 \pi R)^{2}} \int d \mathbf{r}_{1} \int d \mathbf{r}_{2} \rho_{2 \mathrm{D}}\left(\mathbf{r}_{1}\right) \rho_{2 \mathrm{D}}\left(\mathbf{r}_{2}\right) \mathcal{K}\left(r_{12}\right) \\
\times \delta\left(R-\left|\mathbf{r}-\mathbf{r}_{1}\right|\right) \delta\left(R-\left|\mathbf{r}-\mathbf{r}_{2}\right|\right),
\end{gathered}
$$

with $\delta(x)$ and $\Theta(x)$ the Dirac-delta and Heaviside functions, respectively. Note that $\eta_{\mathrm{HD}}(\mathbf{r})$ is just the local packing fraction, while $N_{\mathrm{HD}}(\mathbf{r})$ is a two-body weighted density defined through the kernel

$$
\mathcal{K}(r)=4 \pi R^{2} x \sin ^{-1}(x) \sqrt{1-x^{2}} \Theta(1-x), \quad x \equiv \frac{r}{2 R} .
$$

We now restrict the degrees of freedom by imposing that the HD centers of mass be located on a circumference of radius $R_{0}$ :

$$
\rho_{2 \mathrm{D}}(r, \phi)=\rho_{1 \mathrm{D}}(\phi) \delta\left(R_{0}-r\right),
$$

where $\rho_{1 \mathrm{D}}(\phi)$ is the $1 \mathrm{D}$ density profile, and substitute Eq. (7) into Eqs. (3)-(5). Then we take the following steps (the details 

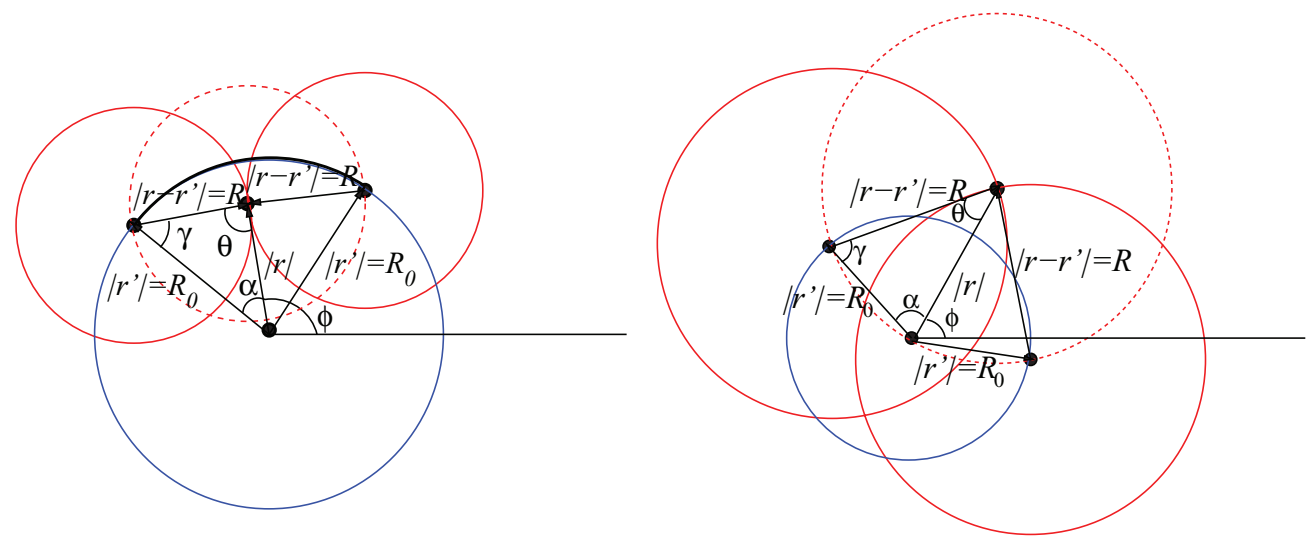

FIG. 1. (Color online) Sketch of the HD configuration on a circle with $R_{0}>R$ (left) and $R_{0}<R$ (right).

of which can be found in Appendix A): (i) A first change of variables $\left(\phi_{i}, r_{i}\right) \rightarrow\left(\phi_{i}, \xi_{i}\right), i=1,2$, with $\xi_{i}=\left|\mathbf{r}-R_{0} \mathbf{u}_{i}\right|$ $\left[\mathbf{u}_{i}=\left(\cos \phi_{i}, \sin \phi_{i}\right)\right.$ are unit vectors] is used to evaluate the weighted densities. As a result they become functions solely of $\phi$ and $r$. (ii) Express the weighted densities as a function of the three angles $\alpha(r), \theta(r)$, and $\gamma(r)$ [the inner angles of the triangle of sides $r, R$, and $R_{0}$; see Fig. 1] and of their derivatives with respect to $r$. (iii) Write the second term of the expression for the free-energy density (2) as a sum of the derivatives of the first term with respect to $\alpha$ and $\phi$. (iv) A second change of variables $(\phi, r) \rightarrow(\phi, \gamma)$ in Eq. (1). (v) Use of the periodicity of the density profile with respect to the azimuthal angle, $\rho(r, \phi)=$ $\rho(r, \phi+2 \pi)$, and integration by parts to finally arrive at the following expression for the excess part of the DF of HDs:

$$
\begin{aligned}
\beta \mathcal{F}_{\mathrm{ex}}^{(\mathrm{HD})}[\rho]= & \frac{R_{0}}{\pi} \int_{0}^{2 \pi} d \phi \int_{0}^{\pi} d \gamma \\
& \times\left\{\Psi(\phi, \alpha(\gamma))+\frac{\partial \Psi}{\partial \alpha}(\phi, \alpha(\gamma)) \theta^{*}(\gamma)\left|\frac{d \alpha}{d \gamma}(\gamma)\right|\right\} .
\end{aligned}
$$

Here the shorthand notations $\Psi(\phi, \alpha)=$ $-n_{0+}(\phi, \alpha) \ln \left[1-\eta_{\mathrm{HD}}(\phi, \alpha)\right] \quad$ and $\quad n_{0+}(\phi, \alpha)=$ $\left[\rho_{1 \mathrm{D}}(\phi+\alpha)+\rho_{1 \mathrm{D}}(\phi-\alpha)\right] / 2$ were used, while the local packing fraction and the angle $\theta^{*}$ are defined as $\eta_{\mathrm{HD}}(\phi, \alpha)=R_{0} \int_{\phi-\alpha}^{\phi+\alpha} d \phi_{1} \rho_{1 \mathrm{D}}\left(\phi_{1}\right)$ and $\theta^{*}=\theta$ if $0 \leqslant \theta \leqslant \pi / 2$, while $\theta^{*}=\pi / 2-\theta$ if $\pi / 2<\theta \leqslant \pi$.

\section{The case $R_{0}>R$}

A look at Fig. 1 allows us to write the following two relations between the angles $\alpha$ and $\gamma$ :

$$
\frac{d \alpha}{d \gamma} \geqslant 0 \text { if } 0<\gamma \leqslant \gamma_{0}, \quad \frac{d \alpha}{d \gamma}<0 \text { if } \gamma_{0}<\gamma \leqslant \pi,
$$

where $\gamma_{0}=\cos ^{-1}\left(R / R_{0}\right), \quad \theta_{0} \equiv \theta\left(\gamma_{0}\right)=\pi / 2, \quad$ and $\quad \alpha_{0} \equiv$ $\alpha\left(\gamma_{0}\right)=\sin ^{-1}\left(R / R_{0}\right)$. After some lengthy calculations, which can be followed in detail in Appendix A 1, we obtain from (8) and (9) the following dimensional crossover:

$$
\beta \mathcal{F}_{\mathrm{ex}}^{(\mathrm{HD})}[\rho] \rightarrow \beta \mathcal{F}_{\mathrm{ex}}^{(\mathrm{CA})}[\rho]=R_{0} \int_{0}^{2 \pi} d \phi \Phi_{1 \mathrm{D}}^{(\mathrm{CA})}(\phi),
$$

with $\Phi_{1 \mathrm{D}}^{\mathrm{CA}}(\phi)$ the 1D Percus free-energy density:

$$
\Phi_{1 \mathrm{D}}^{(\mathrm{CA})}(\phi) \equiv \Psi\left(\phi, \alpha_{0}\right)=-n_{0}^{(\mathrm{CA})}(\phi) \ln \left[1-\eta_{\mathrm{CA}}(\phi)\right],
$$

and with the corresponding weighted densities

$$
\begin{gathered}
n_{0}^{(\mathrm{CA})}(\phi)=n_{0+}\left(\phi, \alpha_{0}\right)=\frac{1}{2}\left[\rho_{1 \mathrm{D}}\left(\phi-\alpha_{0}\right)+\rho_{1 \mathrm{D}}\left(\phi+\alpha_{0}\right)\right] \\
\eta_{\mathrm{CA}}(\phi)=\eta_{\mathrm{HD}}\left(\phi, \alpha_{0}\right)=R_{0} \int_{\phi-\alpha_{0}}^{\phi+\alpha_{0}} d \phi^{\prime} \rho_{1 \mathrm{D}}\left(\phi^{\prime}\right)
\end{gathered}
$$

Here the index "CA" means that these densities are evaluated on a circular arc of length $2 R_{0} \alpha_{0}$. Then we have proved that the excess part of the HD free-energy functional reduces to that of hard CAs.

\section{The case $R_{0}<R$}

In this case we always have

$$
\frac{d \alpha}{d \gamma}<0, \quad \forall \gamma, \quad 0 \leqslant \theta<\pi / 2
$$

(see Fig. 1). After some algebra (described in detail in Appendix A 2), we obtain from (8) and (14) the following dimensional crossover:

$$
\beta \mathcal{F}_{\mathrm{ex}}^{(\mathrm{HD})}[\rho] \rightarrow \beta \mathcal{F}_{\mathrm{ex}}^{(\mathrm{CA})}[\rho]=\eta_{\mathrm{CA}}+\left(1-\eta_{\mathrm{CA}}\right) \ln \left(1-\eta_{\mathrm{CA}}\right),
$$

corresponding to the exact zero-dimensional (OD) functional of CA (such that at most one arc can exist on the circle). The mean number of particles is

$$
\eta_{\mathrm{CA}} \equiv R_{0} \int_{-\pi}^{\pi} d \phi^{\prime} \rho_{1 \mathrm{D}}\left(\phi^{\prime}\right)=2 \pi R_{0} \bar{\rho}_{1 \mathrm{D}}
$$

with $\bar{\rho}_{1 \mathrm{D}}$ the mean number density over the circle of radius $R_{0}$.

\section{B. Functional for CR}

We now define a collection of CRs, each consisting of two parallel curved edges formed by circular arcs of length $2 R_{0} \alpha_{0}$ and two parallel straight lines of length $L$ perpendicular to the former. The centers of mass of the CRs are located on a $2 \mathrm{D}$ cylindrical surface of radius $R_{0}$. The density profile will be $\rho_{2 \mathrm{D}}(\phi, z)$, where $z$ is the coordinate along the cylinder axis and $\phi$ is the azimuthal angle. The functional for this model will be obtained in the two cases. 


\section{The case $R_{0}>R$}

First, we define the local packing fraction as

$$
\eta_{\mathrm{CR}}(\phi, z)=R_{0} \int_{\phi-\alpha_{0}}^{\phi+\alpha_{0}} d \phi^{\prime} \int_{z-L / 2}^{z+L / 2} d z^{\prime} \rho_{2 \mathrm{D}}\left(\phi^{\prime}, z^{\prime}\right),
$$

and the weighted density

$$
n_{1, \perp}^{(\mathrm{CR})}(\phi, z)=\frac{1}{2} \int_{z-L / 2}^{z+L / 2} d z^{\prime}\left[\rho_{2 \mathrm{D}}\left(\phi-\alpha_{0}, z^{\prime}\right)+\rho_{2 \mathrm{D}}\left(\phi+\alpha_{0}, z^{\prime}\right)\right] .
$$

Let us write the modified 1D Percus free-energy density:

$$
\tilde{\Phi}_{1 \mathrm{D}}(\phi, z)=-n_{1, \perp}^{(\mathrm{CR})}(\phi, z) \ln \left[1-\eta_{\mathrm{CR}}(\phi, z)\right] .
$$

Note that this is in fact a local free-energy density corresponding to the CA fluid. Now the CR free-energy density can be calculated by applying a differential operator $\partial / \partial L$ to the modified 1D free-energy density [17]:

$$
\Phi_{2 \mathrm{D}}^{(\mathrm{CR})}(\phi, z)=\frac{\partial}{\partial L} \tilde{\Phi}_{1 \mathrm{D}}(\phi, z)
$$

which results in

$$
\begin{aligned}
\Phi_{2 \mathrm{D}}^{(\mathrm{CR})}(\phi, z)= & -n_{0}^{(\mathrm{CR})}(\phi, z) \ln \left[1-\eta_{\mathrm{CR}}(\phi, z)\right] \\
& +\frac{n_{1, \perp}^{(\mathrm{CR})}(\phi, z) n_{1, \|}^{(\mathrm{CR})}(\phi, z)}{1-\eta_{\mathrm{CR}}(\phi, z)},
\end{aligned}
$$

where we have defined

$$
\begin{aligned}
n_{0}^{(\mathrm{CR})}(\phi, z)= & \frac{\partial}{\partial L} \tilde{n}_{1, \perp}^{(\mathrm{CR})}(\phi, z) \\
= & \frac{1}{4}\left[\rho_{2 \mathrm{D}}\left(\phi_{-}, z_{-}\right)+\rho_{2 \mathrm{D}}\left(\phi_{-}, z_{+}\right)+\rho_{2 \mathrm{D}}\left(\phi_{+}, z_{-}\right)\right. \\
& \left.+\rho_{2 \mathrm{D}}\left(\phi_{+}, z_{+}\right)\right], \\
n_{1, \|}^{(\mathrm{CR})}(\phi, z)= & \frac{\partial}{\partial L} \eta_{\mathrm{CR}}(\phi, z) \\
= & \frac{R_{0}}{2} \int_{\phi_{-}}^{\phi_{+}} d \phi^{\prime}\left[\rho_{2 \mathrm{D}}\left(\phi^{\prime}, z_{-}\right)+\rho_{2 \mathrm{D}}\left(\phi^{\prime}, z_{+}\right)\right],
\end{aligned}
$$

and $z_{ \pm}=z \pm L / 2, \phi_{ \pm}=\phi \pm \alpha_{0}$. Then the excess CR freeenergy functional can be calculated as

$$
\beta \mathcal{F}_{\mathrm{ex}}^{(\mathrm{CR})}[\rho]=R_{0} \int_{0}^{2 \pi} d \phi \int_{-\infty}^{\infty} d z \Phi_{2 \mathrm{D}}^{(\mathrm{CR})}(\phi, z) .
$$

Note that (21) is equivalent to the excess free-energy density of a fluid of parallel hard rectangles (PHRs) of length $L$ and width $\sigma=2 R_{0} \alpha_{0}$ which in turn coincides with that of the parallel hard square (PHS) fluid after scaling one of the edge lengths [17]. Also note that, taking the limit $R_{0} / R \rightarrow \infty$, changing the variable $\phi$ to $x \equiv R_{0} \phi$, and setting $\sigma=2 R_{0} \alpha_{0}$, we obtain from (24) the excess part of the density functional of PHRs on a flat surface of edge lengths $\sigma$ and $L$ given in Ref. [17]. Thus the present functional has the same degree of exactness as that for the PHS fluid. As was shown in Ref. [20], the minimization of the latter gives a phase behavior in which the columnar and crystalline phases both bifurcate from the uniform fluid branch at $\eta \approx 0.54$, with the columnar phase being the stable phase (although the difference between free energies is very small) up to $\eta \approx 0.73$, where a weak first-order columnar-to-crystal transition occurs. Single-speed molecular dynamics simulations of PHSs show a second-order melting transition at $\eta \approx 0.79$ [21], similar to the above given value. However, columnar ordering was not found in the simulations.

\section{The case $R_{0}<R$}

If $R_{0}<R$ we define a $1 \mathrm{D}$ local packing fraction as

$$
\eta_{\mathrm{CR}}(z)=R_{0} \int_{-\pi}^{\pi} d \phi \int_{z-L / 2}^{z+L / 2} d z^{\prime} \rho_{2 \mathrm{D}}\left(\phi^{\prime}, z^{\prime}\right)
$$

and a modified OD free-energy density as

$$
\tilde{\Phi}_{0 \mathrm{D}}^{(\mathrm{CR})}(z)=\eta_{\mathrm{CR}}(z)+\left[1-\eta_{\mathrm{CR}}(z)\right] \ln \left[1-\eta_{\mathrm{CR}}(z)\right] .
$$

The CR free-energy density for this case can be obtained by using the differential operator $\partial / \partial L$ applied to the modified OD free-energy density [17]:

$$
\frac{\partial}{\partial L} \tilde{\Phi}_{0 \mathrm{D}}^{(\mathrm{CR})}(z)=R_{0} \Phi_{1 \mathrm{D}}^{(\mathrm{CR})}(z)=-R_{0} n_{0}^{(\mathrm{CR})}(z) \ln \left[1-\eta_{\mathrm{CR}}(z)\right],
$$

where

$$
\begin{aligned}
n_{0}^{(\mathrm{CR})}(z) & =R_{0}^{-1} \frac{\partial}{\partial L} \eta_{\mathrm{CR}}(z) \\
& =\frac{1}{2} \int_{-\pi}^{\pi} d \phi^{\prime}\left[\rho_{2 \mathrm{D}}\left(\phi^{\prime}, z_{-}\right)+\rho_{2 \mathrm{D}}\left(\phi^{\prime}, z_{+}\right)\right] .
\end{aligned}
$$

The excess free-energy functional is now

$$
\beta \mathcal{F}_{\mathrm{ex}}^{(\mathrm{CR})}[\rho]=2 \pi R_{0} \int_{-\infty}^{\infty} d z \Phi_{1 \mathrm{D}}^{(\mathrm{CR})}(z)
$$

\section{Confined hard cylinders}

The free energy of a collection of CRs can be obtained from two different routes. In the previous two sections we used a two-step process: First, the HD fluid was projected on the circumference of a circle, giving the functional for $\mathrm{CA}$. This in turn was developed along $z$ to give the free energy for CR. There is another possible route which starts from the three-dimensional (3D) functional for hard cylinders (HCs). The $\mathrm{HC}$ excess free energy is

$$
\beta \mathcal{F}_{\mathrm{ex}}^{(\mathrm{HC})}[\rho]=\int d z \int d \phi \int d r r \Phi_{3 \mathrm{D}}^{(\mathrm{HC})}(r, \phi, z),
$$

with $\Phi_{3 \mathrm{D}}^{(\mathrm{HC})}(r, \phi, z)$ the excess free-energy density [18,22]. Now we confine the $\mathrm{HC}$ on a plane perpendicular to the cylinder axes at $z=0$ by choosing the $3 \mathrm{D}$ density profile $\rho_{3 \mathrm{D}}(r, \phi, z)=\rho_{2 \mathrm{D}}(r, \phi) \delta(z)$. Inserting this into (30), we obtain an excess free energy

$$
\beta \mathcal{F}_{\mathrm{ex}}^{(\mathrm{HD})}[\rho]=\int d \phi \int \operatorname{drr} \Phi_{2 \mathrm{D}}^{(\mathrm{HD})}(r, \phi),
$$

where, due to the dimensional-crossover consistency, $\Phi_{2 \mathrm{D}}^{(\mathrm{HD})}(r, \phi)$ is given by Eq. (2), i.e., coincides with the HD freeenergy density. Selecting now $\rho(r, \phi, z)=\rho(\phi, z) \delta\left(R_{0}-r\right)$ (i.e., confining the $\mathrm{HC}$ on a $2 \mathrm{D}$ cylindrical surface of radius $R_{0}$ ) and inserting it into Eq. (30), we obtain, for $R_{0}>R$,

$$
\beta \mathcal{F}_{\mathrm{ex}}^{(\mathrm{CR})}[\rho]=R_{0} \int_{0}^{2 \pi} d \phi \int_{-\infty}^{\infty} d z \Phi_{2 \mathrm{D}}^{(\mathrm{CR})}(\phi, z),
$$




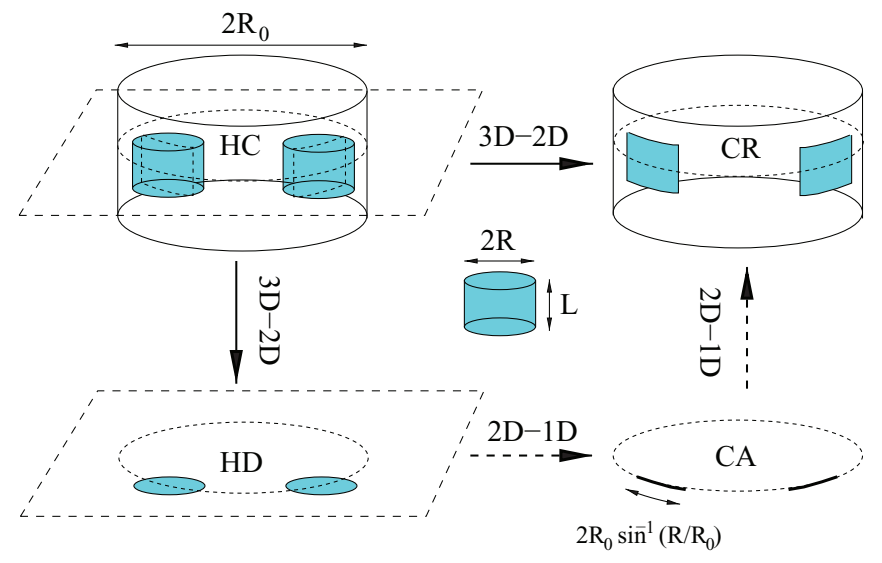

FIG. 2. (Color online) Dimensional crossover of HCs of radius $R$ and length $L$ confined on a cylindrical surface of radius $R_{0}$. This $3 \mathrm{D} \rightarrow 2 \mathrm{D}$ crossover becomes HC into CR. Also shown is the $3 \mathrm{D} \rightarrow$ 2D crossover when the HCs are confined on a flat surface. This crossover becomes HC into HD. Finally the $2 \mathrm{D} \rightarrow 1 \mathrm{D}$ crossover from $\mathrm{HD}$ or CR when both are confined on a circumference of radius $R_{0}$ results in CAs of length $2 R_{0} \sin ^{-1}\left(R / R_{0}\right)$ (the arc of closest approach between two HDs). Dashed arrows indicate the route to obtain the FMF for CR.

where $\Phi_{2 \mathrm{D}}^{(\mathrm{CR})}(\phi, z)$ is the free-energy density of curved rectangles (20). When $R_{0}<R$ we obtain

$$
\beta \mathcal{F}_{\mathrm{ex}}^{(\mathrm{CR})}[\rho]=2 \pi R_{0} \int_{-\infty}^{\infty} d z \Phi_{1 \mathrm{D}}^{(\mathrm{CR})}(z)
$$

where $\Phi_{1 \mathrm{D}}^{(\mathrm{CR})}(z)$ is the free-energy density (27).

We sketch in Fig. 2 the dimensional crossovers involved in the preceding discussion.

\section{PHASE BEHAVIOR OF CR}

In this section we analyze the phase behavior of the CR fluid predicted by the functional derived in the previous section, in the nontrivial case $R_{0}>R$. We start by presenting the numerical treatment in Sec. III A and then show the results in Sec. IV.

\section{A. Minimization technique}

The total free-energy density functional (ideal plus excess parts) per unit of area is

$$
\frac{\beta \mathcal{F}^{(\mathrm{CR})}[\rho]}{2 \pi R_{0} \mathcal{L}}=\frac{1}{2 \pi d} \int_{0}^{2 \pi} d \phi \int_{0}^{d} d z\left[\Phi_{\mathrm{id}}^{(\mathrm{CR})}(\phi, z)+\Phi_{2 \mathrm{D}}^{(\mathrm{CR})}(\phi, z)\right]
$$

where $\mathcal{L}$ is the system length along $z$ and

$$
\Phi_{\mathrm{id}}^{(\mathrm{CR})}(\phi, z)=\rho_{2 \mathrm{D}}(\phi, z)\left[\log \rho_{2 \mathrm{D}}(\phi, z)-1\right]
$$

is the ideal part. The excess contribution is given by Eq. (21). The possible ordered phases in the system are smectic (S), columnar (C), and crystal (K) (sketched in Fig. 3). A general density profile will be periodic in $z$ (with period $d$ ) and $\phi$ (with period $2 \pi)$, i.e., $\rho_{2 \mathrm{D}}(\phi+2 \pi, z+d)=\rho_{2 \mathrm{D}}(\phi, z)$, and a convenient representation is a double Fourier expansion

$$
\rho_{2 \mathrm{D}}(\phi, z)=\rho_{0}\left[1+\sum_{(k, m) \neq(0,0)} s_{k m} \cos \left(k N_{0} \phi\right) \cos (q m z)\right],
$$

where $\rho_{0}$ is the mean density $\rho_{0}=$ $(2 \pi d)^{-1} \int_{0}^{2 \pi} d \phi \int_{0}^{d} d z \rho_{2 \mathrm{~d}}(z, \phi), \quad\left\{s_{k m}\right\}$ are the Fourier amplitudes, and $q=2 \pi / d$ is the wave vector along $z$. Note that $s_{k m}=0 \forall k \neq 0$ corresponds to the $\mathrm{S}$ phase, while $s_{k m}=0$ $\forall m \neq 0$ implies a $C$ phase. In the latter phase the obvious periodicity $\rho_{2 \mathrm{D}}(\phi)=\rho(\phi+2 \pi)$ must be supplemented with a periodicity $\rho_{2 \mathrm{D}}\left(\phi+\phi_{0}\right)=\rho_{2 \mathrm{D}}(\phi)$, with $\phi_{0}=2 \pi / N_{0}$ the period; here the average position of the columns would be located on the vertices of a $N_{0}$-sided regular polygon. $N_{0}$ is an integer in the interval $2-\left[\pi / \alpha_{0}\right]$, with $[x]$ the integer part of $x$, and $\alpha_{0}=\sin ^{-1}\left(R / R_{0}\right)$. As an example, if $R_{0}=2 R$, we have $N_{0}^{(\max )}=6$, a value that can be reached only at close packing.

Using the above Fourier expansion, the weighted densities (17), (18) and (22), (23) can be calculated from the expressions given in Appendix B [see Eqs. (B2)-(B4)]. The strategy followed to minimize the functional (34) was to use a truncated Fourier expansion in terms of the amplitudes $\left\{s_{k m}\right\}$ for $0 \leqslant$ $k, m \leqslant M$ (with $M$ selected in such a way as to guarantee an adequate description of the density profile) and then minimize with respect to these amplitudes and the period $d$. We used a conjugate-gradient method to numerically implement the (a)

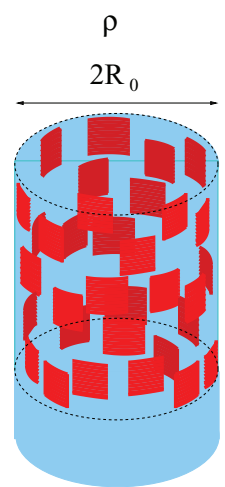

(b)

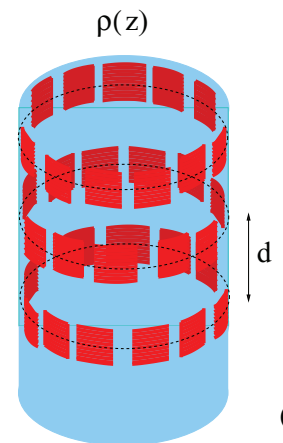

(c)

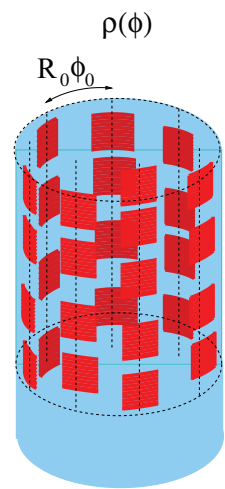

(d)

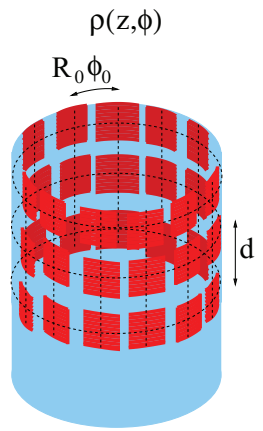

FIG. 3. (Color online) Phases of CRs on a cylindrical surface of radius $R_{0}$ : (a) N, (b) S, (c) C, and (d) K. The lattice parameters and the variables of which the density profiles depend are correspondingly labeled. 

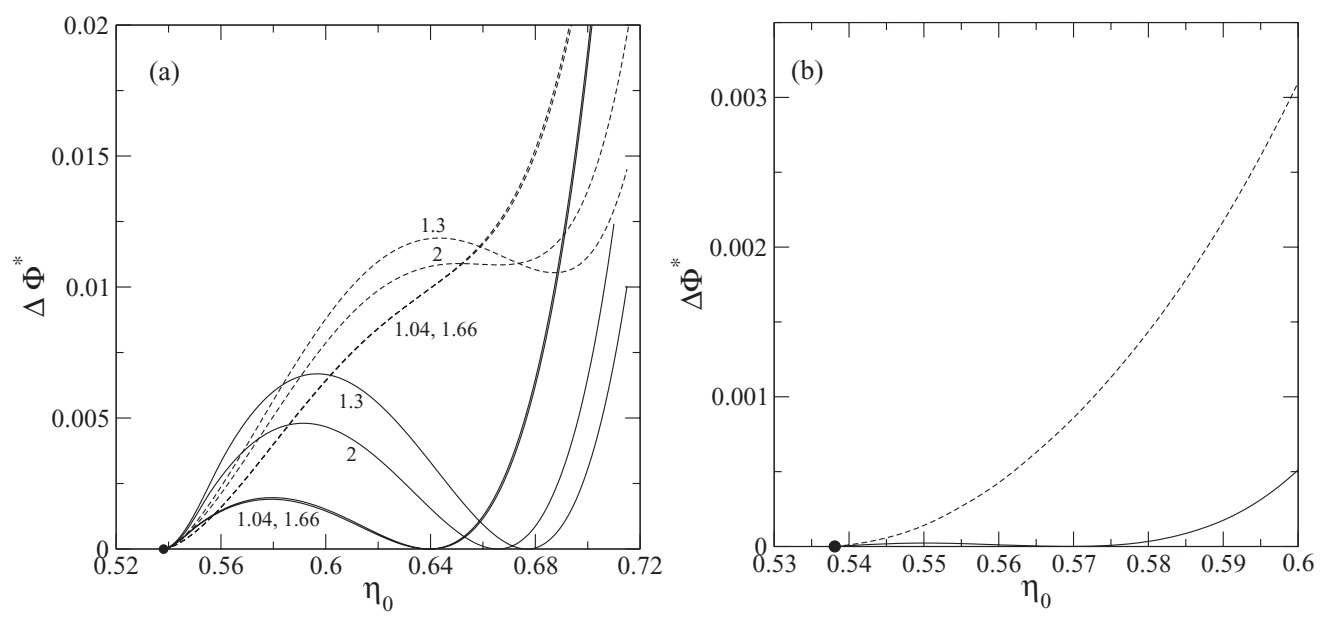

FIG. 4. (a) Free-energy differences $\Delta \Phi_{\mathrm{C}, \mathrm{K}}^{*}$ (see the text for the definition) between the $\mathrm{C}$ and $\mathrm{S}$ (solid lines) phases and $\mathrm{K}$ and $\mathrm{S}$ phases (dashed lines) and for different values of $R_{0} / R$; in (a) they are equal to 1.04, 1.3, 1.66, and 2. Different lines are labeled with their corresponding values of $R_{0} / R$. The number of columns $N_{0}$ in the C phases are 2, 3, 4, and 5, respectively. In (b) $R_{0} / R=1.05$ and $N_{0}=2$. The crystalline phase in (b) was calculated through the free-energy minimization with respect to the Fourier amplitudes of the density-profile Fourier expansion.

minimization. All the integrals were calculated using Legendre quadratures with as many roots as necessary to guarantee numerical errors in the amplitudes below $10^{-7}$.

As shown in the next section, the unstable or metastable character of the crystalline phase is difficult to obtain by direct minimization, so in this case we chose to parametrize the density profile as a sum of Gaussians:

$$
\begin{aligned}
\rho_{2 \mathrm{D}}(\phi, z) & =(1-v) \zeta_{0 \perp}(\phi) \zeta_{0 \|}(z), \\
\zeta_{0 \perp}(\phi) & =\left(\frac{\Lambda_{\perp}}{\pi}\right)^{1 / 2} \sum_{k} \exp \left[-\Lambda_{\perp} R_{0}^{2}\left(\phi-k \phi_{0}\right)^{2}\right], \\
\zeta_{0 \|}(z) & =\left(\frac{\Lambda_{\|}}{\pi}\right)^{1 / 2} \sum_{k} \exp \left[-\Lambda_{\|}(z-k d)^{2}\right],
\end{aligned}
$$

where $\Lambda_{\perp}\left(2 R_{0} \alpha_{0}\right)^{2}=\Lambda_{\|} L^{2}=\Lambda$ is the scaled Gaussian parameter and $v$ the fraction of vacancies. Within this approximation, the total free energy per unit of area, in reduced units, can be computed from the expression given in Appendix B [see Eq. (B5)]. We minimized (B5) with respect to $\Lambda$ and $\nu$. The mean packing fraction can be computed as $\eta_{0}=\frac{(1-v) a}{R_{0} \phi_{0} d}$ (with $a=2 R_{0} \alpha_{0} L$ the particle area) and thus for fixed $\eta_{0}$ and $v$ we can compute the period $d$ from the latter equation. The integrals of Eq. (B5) were evaluated using a GaussHermite quadrature while the minimization was carried out using the Newton-Raphson method with supplied numerical derivatives.

\section{RESULTS}

We minimized (34) using the Fourier expansion of the density profile (36) for the $\mathrm{S}$ and $\mathrm{C}$ phases and for four different values of $R_{0} / R=1.04,1.30,1.66$, and 2.00. The results are shown in Fig. 4(a) in which we plot the difference between the free energies per unit area corresponding to the $\mathrm{C}$ and $\mathrm{S}$ phases, i.e., $\Delta \Phi_{\mathrm{C}}^{*}=\Phi_{\mathrm{C}}^{*}-\Phi_{\mathrm{S}}^{*}$ (with $\Phi^{*}=\frac{\beta \mathcal{F}\left[\rho_{\mathrm{eq}}\right] a}{2 \pi R_{0} \mathcal{L}}$ ), as a function of the mean packing fraction $\eta_{0}$. For the $\mathrm{C}$ phase the number of columns for each value of $R_{0} / R$ are $N_{0}=2,3,4$, and
5 , respectively. As can be seen, $\Delta \Phi_{\mathrm{C}}^{*} \geqslant 0$ always, implying that the $\mathrm{S}$ phase is the most stable phase. This behavior can be understood if we take into account that the $\mathrm{C}$ period in reduced units is $\phi_{0}^{*}=\phi_{0} R_{0} /\left(2 \alpha_{0} R_{0}\right)=\pi /\left(N_{0} \alpha_{0}\right)$ which in turn is different from that of PHRs (remember that the excess part of the free-energy density of CRs can be obtained from that of PHRs by the mapping $\sigma \rightarrow 2 \alpha_{0} R_{0}$, with $\sigma$ the width of the rectangle); the latter is calculated by minimizing the free energy with respect to the period. Note that, for PHRs, the $\mathrm{C}$ and $\mathrm{S}$ phases have the same free energies (the PHRs can be obtained by scaling the PHSs along one direction). However, in the present case the value of $\phi_{0}^{*}$ is imposed once we fix $R_{0} / R$ and the number of columns $N_{0}$, the latter being dictated by the commensuration of a CA of length $2 \alpha_{0} R_{0}$ in a circle of perimeter $2 \pi R_{0}$, i.e., $2 \leqslant N_{0} \leqslant\left[\pi / \alpha_{0}\right]$. In a case when different values of $N_{0}$ are possible, we select the one which minimizes the free energy. It is interesting to note that $\Delta \Phi^{*}=0$ both at the bifurcation point $\eta_{0}^{*}$ and at that value of $\eta_{0}$ for which the periods in reduced units of CRs $\left(\phi_{0}^{*}\right)$ and of hard parallel rectangles (HPRs) on a plane $\left(d_{\mathrm{HPR}}^{*} \equiv d_{\mathrm{HPR}} / \sigma\right)$ are exactly the same. Finally, from Fig. 4(a), we can see that $\Delta \Phi_{\mathrm{c}}^{*}$ for the cases $R_{0} / R=1.04\left(N_{0}=2\right)$ and $1.66\left(N_{0}=4\right)$ are indistinguishable from each other. The reason behind this behavior is that the commensuration numbers $\pi /\left(N_{0} \alpha_{0}\right)$ [ratio between the perimeter of the circle $\left(2 \pi R_{0}\right)$ and the total length of the $N_{0}$ arcs of length $2 R_{0} \alpha_{0}$ ] are approximately equal in both cases (1.2153 and 1.2148, respectively).

In the same figure we also plot $\Delta \Phi_{\mathrm{K}}^{*}=\Phi_{\mathrm{K}}^{*}-\Phi_{\mathrm{S}}^{*}$, i.e., the difference between the free energies per unit volume of the $\mathrm{K}$ and $\mathrm{S}$ phases. For this case we have used the Gaussian parametrization (37) and minimized (B5) with respect to $\Lambda$ and $d^{*}$ [we tried to use the Fourier expansion (36) with $\mathrm{K}$ symmetry, but the numerical algorithm converged to a solution with $\mathrm{C}$ or $\mathrm{S}$ symmetry always, except for those values of $\eta_{0}$ close enough to the bifurcation point, an example of which is shown in Fig. 4(b)]. In Fig. 4(a) we see that the $\mathrm{K}$ phase has a larger free energy compared to the $S$ phase. However, now $\Delta \Phi_{\mathrm{K}}^{*}$ does not touch the $\eta_{0}$ axis tangentially, because the $\mathrm{PHR}$ free energies corresponding to the $\mathrm{C}$ (or $\mathrm{S}$ ) and $\mathrm{K}$ phases 

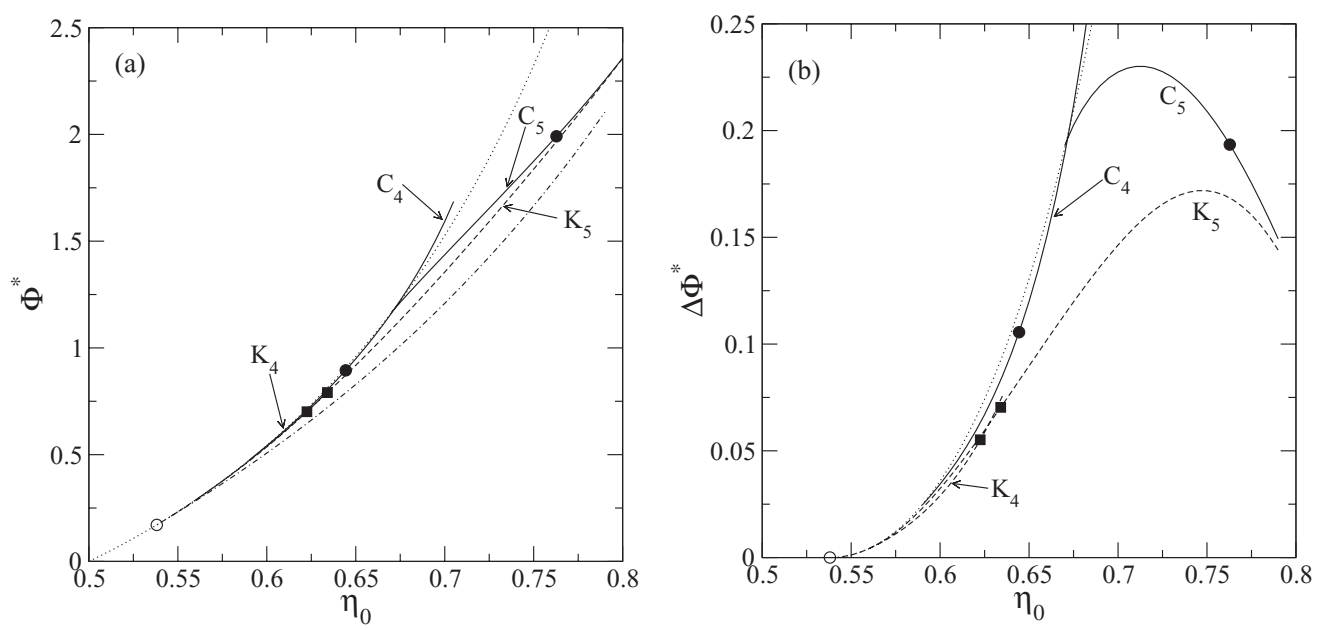

FIG. 5. (a) Free energies per unit of area [we plot $\left.\Phi^{*}+\eta_{0} \ln (a)\right]$ of $\mathrm{N}$ (dotted), $\mathrm{S}$ (dotted-dashed), $\mathrm{C}_{4}$ and $\mathrm{C}_{5}$ (solid lines correspondingly labeled), and $\mathrm{K}_{4}$ and $\mathrm{K}_{5}$ (dashed lines correspondingly labeled) phases as a function of $\eta_{0}$ for $R_{0} / R=1.8$. The open circle indicates the $\mathrm{N}-\mathrm{S}$ bifurcation point. Circles indicate the metastable $\mathrm{C}_{4}-\mathrm{C}_{5}$ coexistence, while squares correspond to the metastable $\mathrm{K}_{4}-\mathrm{K}_{5}$ coexistence. (b) Free-energy density differences between the $\mathrm{S}$ and all the phases shown in (a). The lines and symbols have the same meaning as in (a).

are not equal, the former being energetically favored up to $\eta_{0} \sim 0.73[20]$.

It is interesting to note that the $\mathrm{N}-\mathrm{S}$ transition exists even for values of $R_{0} / R \gtrsim 1$. As already shown, for $R_{0} / R<1$ this transition is absent since the free-energy functional is in fact the 1D functional for hard rods (Percus functional).

Now we present the results for $R_{0} / R=1.8$, a value for which the free-energy branches of the $\mathrm{C}$ phases with $N_{0}=4$ $\left(\mathrm{C}_{4}\right)$ and $5\left(\mathrm{C}_{5}\right)$ columns intersect at some value of $\eta_{0}$. These free energies are plotted in Fig. 5. The $\mathrm{C}_{4}$ and $\mathrm{C}_{5}$ branches are above those of the $\mathrm{K}_{4,5}$ and $\mathrm{S}$ phases. This result could

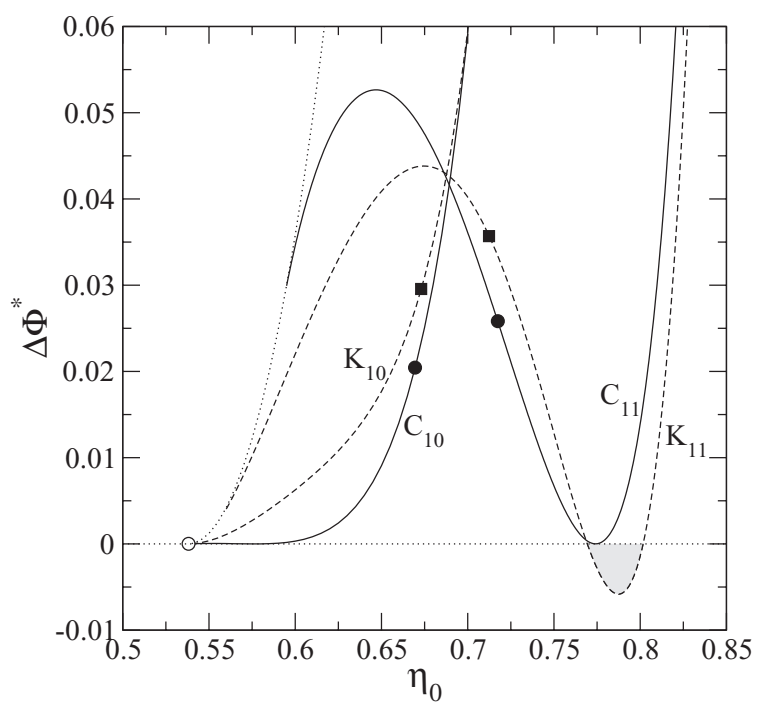

FIG. 6. Free-energy density differences between the $\mathrm{S}$ and the $\mathrm{N}$ (dotted), $\mathrm{C}_{10}$ and $\mathrm{C}_{11}$ (solid lines correspondingly labeled), and $\mathrm{K}_{10}$ and $K_{11}$ (dashed lines correspondingly labeled) phases as a function of the mean packing fraction $\eta_{0}$ for $R_{0} / R=4$. The open circle indicates the N-S bifurcation point. The circles and squares show the $\mathrm{C}_{10}-\mathrm{C}_{11}$ and $\mathrm{K}_{10}-\mathrm{K}_{11}$ metastable coexistences, respectively. The gray region shows the packing fraction interval in which the $\mathrm{K}_{11}$ has a lower free energy than the $S$ phase. change in a confined binary fluid of HCs with different lengths. For dissimilar enough lengths, the $\mathrm{S}$ and $\mathrm{K}$ phases would be energetically unfavored with respect to the $\mathrm{C}_{N_{0}}$ phases. In this situation a $\mathrm{C}_{4}-\mathrm{C}_{5}$ transition could take place as $\eta_{0}$ increases. For some higher values of $R_{0} / R$ we will find a cascade of $\mathrm{C}_{N_{0}-1} \rightarrow \mathrm{C}_{N_{0}}$ transitions for different values of $N_{0}$ because multiple values of $N_{0}$ fulfill the constraint $2 \leqslant N_{0} \leqslant\left[\pi / \alpha_{0}\right]$.

Finally, we have calculated all the energy branches of stable or metastable phases for $R_{0} / R=4$, which are plotted in Fig. 6 . For this case there are two columnar, $\mathrm{C}_{10}$ and $\mathrm{C}_{11}$, and two crystalline, $\mathrm{K}_{10}$ and $\mathrm{K}_{11}$, phases which are metastable with respect to the $\mathrm{Sm}$ phase (except the $\mathrm{K}_{11}$ phase, which is stable in a small range of $\eta_{0}$ ). We show in Fig. 6 the metastable $\mathrm{C}_{10}-\mathrm{C}_{11}$ and $\mathrm{K}_{10}-\mathrm{K}_{11}$ coexistences, using different symbols. Note that the coexistence gaps decrease with respect to the case $R_{0} / R=1.8$, a trend that should be confirmed if the value $R_{0} / R$ were to be increased even more. It is interesting to note that there is a relatively small interval of packing fractions (dashed region in the figure) where the $K_{11}$ phase becomes reentrant. This interval should increase for higher values of $R_{0} / R$ and, in fact, in the limit $R_{0} / R \rightarrow \infty$ (the HPR limit), the $\mathrm{K}$ phase becomes stable for $\eta \gtrsim 0.73$ [20].

\section{DENSITY FUNCTIONAL OF SPHERICAL LENSES}

In principle the same dimensional-crossover procedure can be implemented on the density functional for HSs of radii $R$ whose centers of mass are restricted to be on a spherical surface of radius $R_{0}$. If the density functional for HSs adequately fulfills the dimensional-crossover property, the resulting functional, that of a fluid consisting of hard spherical lenses (SLs) [obtained from the intersection between the spherical surface and a cone with vertex at the origin and with solid angle equal to $4 \pi \sin ^{2}\left(\alpha_{0} / 2\right)=2 \pi[1-$ $\left.\sqrt{1-\left(R / R_{0}\right)^{2}}\right]$ ], should coincide, when $R_{0} / R>1$, with the 2D functional for HDs [Eq. (2)] where the density profile this time is a periodic function of the spherical angles $\rho(\phi, \theta)=$ $\rho(\phi+2 \pi, \theta), \rho(\phi, \theta)=\rho(\phi, \theta+2 \pi)$ and the semilength of 
the SLs is equal to $R_{0} \alpha_{0}=R_{0} \sin ^{-1}\left(R / R_{0}\right)$. We present in Appendix $C$ the explicit expressions for the weighted densities of SLs. With the present tool, the study of the freezing of HSs on a spherical surface could be carried out. An interesting point to be studied is how the symmetry of the crystalline structure, and the presence of defects and vacancies, could change with the ratio $R_{0} / R$.

\section{CONCLUSIONS}

The present work followed two motivations. The first was to illustrate the dimensional-crossover criterion as a powerful projection tool to obtain a DF for hard particles with centers of mass constrained to be on a particular (in the present case cylindrical) surface from a given DF which fulfills this criterion. The second motivation was the study of the phase behavior of CRs (the particles obtained by projecting the centers of mass of HCs onto a cylindrical surface). We show that surface curvature has a profound impact on the stability of the liquid-crystal phases of curved rectangles as compared with the planar case. The projection method is quite general and can be used to obtain DFs for other particle geometries and other surfaces. As an example, we proposed in Sec. V a DF for spherical lenses, i.e., the particles obtained when the centers of mass of HSs are placed on an external spherical surface.

During the derivation of DF for CRs we proved the dimensional-crossover properties of the FMF of HDs of radii $R$ when their centers of mass are constrained to be on a circumference of radius $R_{0}$. Depending on the ratio $R_{0} / R$ the original 2D density functional reduces to the 1D Percus functional (when $\left.R_{0} / R>1\right)$ or to the $0 \mathrm{D}$ functional $\left(R_{0} / R<1\right)$, which are both exact limits. From these dimensionally reduced functionals, using the dimensional expansion procedure, we derive the density functional for CRs moving on a cylindrical surface of radius $R_{0}$. We show that this functional is equivalent to that of PHRs on a flat surface with the edge lengths of the particles being $\sigma=2 R_{0} \sin ^{-1}\left(R / R_{0}\right)$ and $L$. We minimized the functional for CRs to get the phase behavior for different values of $R_{0} / R$. When $R_{0} / R>1$ we obtain that the most stable phase is $\mathrm{S}$, as compared to the $\mathrm{C}$ or $\mathrm{K}$ phases, except for some relatively large values of $R_{0} / R$ and small density intervals in which we find a reentrant $\mathrm{K}$ phase. We find also metastable $\mathrm{C}_{n-1}-\mathrm{C}_{n}$ or $\mathrm{K}_{n-1}-\mathrm{K}_{n}$ transitions related to the commensuration between the particle width and the perimeter of the cylindrical surface. These transitions could become stable when the confined HCs have different lengths (it is well known that the length polydispersity destroys the $\mathrm{S}$ ordering). When $R_{0} / R<1$ the density functional of CRs coincides with the 1D Percus functional, so that the system does not exhibit any phase transition. We are presently performing Monte Carlo (MC) simulations of CRs with the aim to compare the phase behavior with that obtained from the present FMF. Note that the latter, being a 2D functional, is not exact.

The present phase behavior points to possible textures that adsorbed molecules (for example proteins) on cylindrical membranes could exhibit. If these molecules are highly anisotropic, highly oriented along the cylinder axis, and interact repulsively with each other, their stable textures should include only nematic and smecticlike configurations for low and high densities, respectively.
The wall of some rod-shaped bacteria grows in the direction of the cylinder axis, keeping the radius approximately constant. The new proteins come from the inside of the cell to the wall. In Ref. [23] the authors explain cell growth by the insertion of these proteins into the wall and their subsequent active diffusion along the perimeter of the cylinder via a dislocation-mediated growth. The model assumes that the proteins form a square lattice on a surface of the cylinder (this is apparently confirmed by experiments). Protein diffusion along the wall perimeter (activated by the cell machinery) departs from some of these dislocations which constitute the source of the new proteins coming from the cell. Our results show that the smecticlike configuration of proteins favors their transversal diffusion. Also the sources of new molecules could be located at any position inside the smectic layers with marginal energy cost associated with the spatial deformation of layers (as they are fluidlike). However, this mechanism makes the cylindrical membrane grow in the transversal direction. Growth along the longitudinal direction is possible by creating new smectic layers and consequently smecticlike defects in the layers. This is an alternative mechanism that might explain the growth of cells whose membranes are constituted by molecules that form smecticlike textures.

\section{ACKNOWLEDGMENTS}

We acknowledge financial support from Comunidad Autónoma de Madrid (Spain) under the R\&D Programme of Activities MODELICO-CM/S2009ESP-1691, and from MINECO (Spain) under Grants No. MOSAICO, No. FIS201022047-C01, and No. FIS2010-22047-C04.

\section{APPENDIX A: 2D $\rightarrow$ 1D,0D DIMENSIONAL CROSSOVER OF HD ON A CIRCLE}

Substitution of (7) into Eq. (3) gives

$n_{0}^{(\mathrm{HD})}(\mathbf{r})=\frac{R_{0}}{2 \pi R} \int_{0}^{2 \pi} d \phi_{1} \delta\left(R-\left|\mathbf{r}-R_{0} \mathbf{u}_{1}\right|\right) \rho_{1 \mathrm{D}}\left(\phi_{1}\right)$,

where $\mathbf{u}_{1}=\left(\cos \phi_{1}, \sin \phi_{1}\right)$ is a unit vector. Here the polar radius $r$ is restricted to be in the interval $\left[\mid R_{0}-\right.$ $\left.R \mid, R_{0}+R\right]$. Using the change of variables $\xi=\mid \mathbf{r}-$ $R_{0} \mathbf{u}_{1} \mid=\sqrt{r^{2}+R_{0}^{2}-2 r R_{0} \cos \left(\phi-\phi_{1}\right)}$, where $\mathbf{r}=r \mathbf{u}, \mathbf{u}=$ $(\cos \phi, \sin \phi)$, and $\mathbf{u} \cdot \mathbf{u}_{1}=\cos \left(\phi-\phi_{1}\right)$, we obtain

$$
\begin{aligned}
n_{0}^{(\mathrm{HD})}(\mathbf{r})= & \frac{1}{2 \pi r} \frac{\left[\rho_{1 \mathrm{D}}(\phi+\alpha(r))+\rho_{1 \mathrm{D}}(\phi-\alpha(r))\right]}{\sqrt{1-\left(\frac{r^{2}+R_{0}^{2}-R^{2}}{2 r R_{0}}\right)^{2}}} \\
& \times \Theta\left(R-\left|r-R_{0}\right|\right),
\end{aligned}
$$

where

$$
\alpha(r) \equiv \cos ^{-1}\left(\frac{r^{2}+R_{0}^{2}-R^{2}}{2 r R_{0}}\right) .
$$

Now we calculate the two-body weighted density $N_{\mathrm{HD}}(\mathbf{r})$ by substituting $\rho_{2 \mathrm{D}}\left(\mathbf{r}_{i}\right)=\rho_{1 \mathrm{D}}\left(\phi_{i}\right) \delta\left(R_{0}-r_{i}\right)$ [which is equivalent to setting $\mathbf{r}_{i}=R_{0} \mathbf{u}_{i}$ with $\left.\mathbf{u}_{i}=\left(\cos \phi_{i}, \sin \phi_{i}\right)\right]$ into Eq. (5). Noting that $r_{12}=\left|\mathbf{r}_{1}-\mathbf{r}_{2}\right|=2 R_{0}\left|\sin \left(\phi_{12} / 2\right)\right|$ and 
using Eq. (6), we obtain

$$
\begin{aligned}
\mathcal{K}\left(r_{12}\right)= & 4 \pi R^{2} \frac{R_{0}}{R}\left|\sin \left(\phi_{12} / 2\right)\right| \sin ^{-1}\left(\frac{R_{0}}{R}\left|\sin \left(\phi_{12} / 2\right)\right|\right) \\
& \times \sqrt{1-\left[\frac{R_{0}}{R} \sin \left(\phi_{12} / 2\right)\right]^{2}} .
\end{aligned}
$$

Using again $\xi_{i}=\left|\mathbf{r}-\mathbf{r}_{i}\right|$, taking into account that $\left|\sin \left(\phi_{12} / 2\right)\right|_{\xi_{\mathrm{i}}=\mathrm{R}}=|\sin \alpha(r)|$, and using (5), we obtain

$$
\begin{aligned}
N_{\mathrm{HD}}(\mathbf{r})= & \frac{R_{0}}{\pi r^{3}} \frac{\rho_{1 \mathrm{D}}(\phi-\alpha(r)) \rho_{1 \mathrm{D}}(\phi+\alpha(r))}{\sqrt{1-\left(\frac{r^{2}+R_{0}^{2}-R^{2}}{2 r R_{0}}\right)^{2}}}\left|R^{2}+r^{2}-R_{0}^{2}\right| \\
& \times \sin ^{-1}\left[\frac{R_{0}}{R} \sqrt{1-\left(\frac{r^{2}+R_{0}^{2}-R^{2}}{2 r R_{0}}\right)^{2}}\right] \\
& \times \Theta\left(R-\left|r-R_{0}\right|\right) .
\end{aligned}
$$

The local packing fraction (4) results in

$$
\begin{aligned}
\eta_{\mathrm{HD}}(\mathbf{r}) & =R_{0} \int_{0}^{2 \pi} d \phi_{1} \rho_{1 \mathrm{D}}\left(\phi_{1}\right) \Theta\left(R-\left|\mathbf{r}-R_{0} \mathbf{u}_{1}\right|\right) \\
& =R_{0} \int_{\phi-\alpha(r)}^{\phi+\alpha(r)} d \phi_{1} \rho_{1 \mathrm{D}}\left(\phi_{1}\right)
\end{aligned}
$$

where we used the fact that the condition $\left|\mathbf{r}-R_{0} \mathbf{u}_{1}\right| \leqslant$ $R$ implies $\left|\phi-\phi_{1}\right| \leqslant \alpha(r)$. The weighted densities can be written in terms of the angle $\alpha(r)$ and the new angles $\theta(r)$ and $\gamma(r)$, defined in Fig. 1:

$$
n_{0}^{(\mathrm{HD})}(\mathbf{r})=\frac{R_{0}}{2 \pi r}\left[\rho_{1 \mathrm{D}}(\phi-\alpha(r))+\rho_{1 \mathrm{D}}(\phi+\alpha(r))\right] \frac{d \gamma}{d r}(r),
$$

$$
\begin{aligned}
N_{\mathrm{HD}}(\mathbf{r})= & \frac{2 R_{0}^{2}}{\pi r} \rho_{1 \mathrm{D}}(\phi-\alpha(r)) \rho_{1 \mathrm{~d}}(\phi+\alpha(r))\left|\frac{d \alpha}{d \gamma}(r)\right| \\
& \times \theta^{*}(r) \frac{d \gamma}{d r}(r),
\end{aligned}
$$

where

$$
\theta^{*}= \begin{cases}\theta, & \text { if } \quad 0 \leqslant \theta \leqslant \pi / 2, \\ \pi-\theta, & \text { if } \pi / 2 \leqslant \theta \leqslant \pi .\end{cases}
$$

The following equation is satisfied:

$$
\begin{aligned}
& \rho_{1 \mathrm{D}}(\phi-\alpha(r)) \rho_{1 \mathrm{D}}(\phi+\alpha(r)) \\
& \quad=\frac{1}{4}\left\{\frac{2}{R_{0}} n_{0+}(\mathbf{r}) \frac{\partial \eta_{\mathrm{HD}}}{\partial \alpha}(\mathbf{r})-\frac{1}{R_{0}^{2}}\left[\frac{\partial \eta_{\mathrm{HD}}}{\partial \phi}(\mathbf{r})\right]^{2}\right\} .
\end{aligned}
$$

This can be derived from the relation

$$
\frac{\partial \eta_{\mathrm{HD}}}{\partial \alpha}(\mathbf{r})=2 R_{0} n_{0+}(\mathbf{r}), \quad \frac{\partial \eta_{\mathrm{HD}}}{\partial \phi}(\mathbf{r})=2 R_{0} n_{0-}(\mathbf{r})
$$

with

$$
n_{0 \pm}(\mathbf{r})=\frac{1}{2}\left[\rho_{1 \mathrm{D}}(\phi+\alpha(r)) \pm \rho_{1 \mathrm{D}}(\phi-\alpha(r))\right] .
$$

Now using the relations

$$
\begin{aligned}
& \frac{2}{R_{0}} \frac{\partial n_{0+}}{\partial \alpha}(\mathbf{r})-\frac{1}{R_{0}^{2}} \frac{\partial^{2} \eta_{\mathrm{HD}}}{\partial \phi^{2}}(\mathbf{r})=0 \\
\frac{n_{0+}(\mathbf{r}) \frac{\partial \eta_{\mathrm{HD}}}{\partial \alpha}(\mathbf{r})}{1-\eta_{\mathrm{HD}}(\mathbf{r})}= & -\frac{\partial}{\partial \alpha}\left\{n_{0+}(\mathbf{r}) \ln \left[1-\eta_{\mathrm{HD}}(\mathbf{r})\right]\right\} \\
& +\frac{\partial n_{0+}}{\partial \alpha}(\mathbf{r}) \ln \left[1-\eta_{\mathrm{HD}}(\mathbf{r})\right], \\
\frac{\left[\frac{\partial \eta_{\mathrm{HD}}}{\partial \phi}(\mathbf{r})\right]^{2}}{1-\eta_{\mathrm{HD}}(\mathbf{r})}= & -\frac{\partial}{\partial \phi}\left\{\frac{\partial \eta_{\mathrm{HD}}}{\partial \phi}(\mathbf{r}) \ln \left[1-\eta_{\mathrm{HD}}(\mathbf{r})\right]\right\} \\
& +\frac{\partial^{2} \eta_{\mathrm{HD}}}{\partial \phi^{2}}(\mathbf{r}) \ln \left[1-\eta_{\mathrm{HD}}(\mathbf{r})\right],
\end{aligned}
$$

we get

$$
\begin{aligned}
\frac{2 n_{0+}(\mathbf{r}) \frac{\partial \eta_{\mathrm{HD}}}{\partial \alpha}(\mathbf{r})}{R_{0}\left[1-\eta_{\mathrm{HD}}(\mathbf{r})\right]}-\frac{\left[\frac{\partial \eta_{\mathrm{HD}}}{\partial \phi}(\mathbf{r})\right]^{2}}{R_{0}^{2}\left[1-\eta_{\mathrm{HD}}(\mathbf{r})\right]} \\
=-\frac{2}{R_{0}} \frac{\partial}{\partial \alpha}\left\{n_{0+}(\mathbf{r}) \ln \left[1-\eta_{\mathrm{HD}}(\mathbf{r})\right]\right\} \\
\quad+\frac{1}{R_{0}^{2}} \frac{\partial}{\partial \phi}\left\{\frac{\partial \eta_{\mathrm{HD}}}{\partial \phi}(\mathbf{r}) \ln \left[1-\eta_{\mathrm{HD}}(\mathbf{r})\right]\right\} .
\end{aligned}
$$

Next we take into account the periodic condition $\eta_{\mathrm{HD}}(r, \phi)=$ $\eta_{\mathrm{HD}}(r, \phi+2 \pi)$ which implies

$$
\int_{0}^{2 \pi} d \phi \frac{\partial}{\partial \phi}\left\{\frac{\partial \eta_{\mathrm{HD}}}{\partial \phi}(\mathbf{r}) \ln \left[1-\eta_{\mathrm{HD}}(\mathbf{r})\right]\right\}=0 .
$$

Equations (A10), (A14), and (A15) can be used to give

$$
\begin{aligned}
& \int_{0}^{2 \pi} d \phi \frac{2 R_{0}^{2}}{\pi} \frac{\rho_{1 \mathrm{D}}(\phi-\alpha(r)) \rho_{1 \mathrm{D}}(\phi+\alpha(r))}{1-\eta_{\mathrm{HD}}(\mathbf{r})} \\
& =-\int_{0}^{2 \pi} d \phi \frac{R_{0}}{\pi} \frac{\partial}{\partial \alpha}\left\{n_{0+}(\mathbf{r}) \ln \left[1-\eta_{\mathrm{HD}}(\mathbf{r})\right]\right\} .
\end{aligned}
$$

Introducing the change of variable $(r, \phi) \rightarrow(\gamma, \phi)$ and using Eqs. (A7), (A8), and (A16), the excess part of the free energy (1) can be rewritten as (8).

\section{The case $R_{0}>R$}

Using (9) we have

$$
\begin{aligned}
& \int_{0}^{\pi} d \gamma \frac{\partial \Psi}{\partial \alpha}[\phi, \alpha(\gamma)] \theta^{*}(\gamma)\left|\frac{d \alpha}{d \gamma}(\gamma)\right|=\int_{0}^{\gamma_{0}} d \gamma \frac{\partial \Psi}{\partial \alpha}[\phi, \alpha(\gamma)][\pi-\theta(\gamma)] \frac{d \alpha}{d \gamma}(\gamma)+\int_{\gamma_{0}}^{\pi} d \gamma \frac{\partial \Psi}{\partial \alpha}[\phi, \alpha(\gamma)] \theta(\gamma)\left[-\frac{d \alpha}{d \gamma}(\gamma)\right] \\
& =[\pi \Psi(\phi, \alpha)]_{\alpha(0)}^{\alpha_{0}}-\underbrace{\int_{0}^{\pi} d \gamma \frac{\partial}{\partial \alpha}[\Psi(\phi, \alpha(\gamma)) \theta(\gamma)] \frac{d \alpha}{d \gamma}(\gamma)}_{=[\Psi(\phi, \alpha) \theta(\alpha)]_{\alpha(0)}^{\alpha(\pi)}=0}+\int_{0}^{\pi} d \gamma \Psi(\phi, \alpha(\gamma)) \frac{d \theta}{d \alpha}(\gamma) \frac{d \alpha}{d \gamma}(\gamma) \\
& =\pi \Psi\left(\phi, \alpha_{0}\right)+\int_{0}^{\pi} d \gamma \Psi(\phi, \alpha(\gamma)) \frac{d \theta}{d \alpha}(\gamma) \frac{d \alpha}{d \gamma}(\gamma)
\end{aligned}
$$


where we have used the change of variable $\int_{\gamma_{1}}^{\gamma_{2}} d \gamma T(\phi, \alpha(\gamma)) \frac{d \alpha}{d \gamma}(\gamma)=\int_{\alpha\left(\gamma_{1}\right)}^{\alpha\left(\gamma_{2}\right)} d \alpha T(\phi, \alpha)$ for a general function $T(\phi, \alpha)$ and also that $\alpha(0)=\alpha(\pi)=0$. Now from (A17) and (8) we can reexpress the excess free energy as

$$
\begin{aligned}
\beta \mathcal{F}_{\mathrm{ex}}^{(\mathrm{HD})}[\rho]= & \frac{R_{0}}{\pi} \int_{0}^{2 \pi} d \phi\left\{\pi \Psi\left(\phi, \alpha_{0}\right)+\int_{0}^{\pi} d \gamma \Psi(\phi, \alpha(\gamma))\right. \\
& \left.\times\left[1+\frac{d \theta}{d \alpha}(\gamma) \frac{d \alpha}{d \gamma}(\gamma)\right]\right\} .
\end{aligned}
$$

Further, since $\alpha+\theta+\gamma=\pi$, we have

$$
d \gamma+\frac{d \theta}{d \alpha} \frac{d \alpha}{d \gamma} d \gamma=d \alpha\left(\frac{d \gamma}{d \alpha}+\frac{d \theta}{d \alpha}\right)=-d \alpha
$$

and

$$
\begin{aligned}
& \beta \mathcal{F}_{\mathrm{ex}}^{(\mathrm{HD})}[\rho] \\
& =\frac{R_{0}}{\pi} \int_{0}^{2 \pi} d \phi\{\pi \Psi\left(\phi, \alpha_{0}\right)-\underbrace{\int_{\alpha(0)}^{\alpha(\pi)} d \alpha \Psi(\phi, \alpha)}_{=0}\} .
\end{aligned}
$$

Let us redefine the weighted densities as in (12) and (13) where the index "CA" means that these densities are those on a circular arc of length $2 R_{0} \alpha_{0}$. Then we have proved that the excess part of the HD free-energy functional reduces to that of hard CA, given by Eq. (10).

\section{The case $R_{0}<R$}

Taking into account (14), Eq. (8) reads

$$
\begin{aligned}
\beta \mathcal{F}_{\mathrm{ex}}^{(\mathrm{HD})}[\rho]= & \frac{R_{0}}{\pi} \int_{0}^{2 \pi} d \phi \int_{0}^{\pi} d \gamma \\
& \times\left\{\Psi(\phi, \alpha(\gamma))-\frac{\partial \Psi}{\partial \alpha}[\phi, \alpha(\gamma)] \theta(\gamma) \frac{d \alpha}{d \gamma}(\gamma)\right\} .
\end{aligned}
$$

Using

$$
\frac{\partial \Psi}{\partial \alpha}(\phi, \alpha) \theta(\gamma)=\frac{\partial}{\partial \alpha}[\Psi(\phi, \alpha) \theta(\gamma)]-\Psi(\phi, \alpha) \frac{d \theta}{d \alpha}(\gamma),
$$

Eq. (A21) becomes

$$
\begin{aligned}
\beta \mathcal{F}_{\mathrm{ex}}^{(\mathrm{HD})}[\rho] & \frac{R_{0}}{\pi} \int_{0}^{2 \pi} d \phi\left\{\int_{0}^{\pi} d \gamma \Psi(\phi, \alpha(\gamma))\left[1+\frac{d \theta}{d \alpha}(\gamma) \frac{d \alpha}{d \gamma}(\gamma)\right]\right. \\
& \left.-\int_{0}^{\pi} d \gamma \frac{d \alpha}{d \gamma}(\gamma) \frac{\partial}{\partial \alpha}[\Psi(\phi, \alpha(\gamma)) \theta(\gamma)]\right\},
\end{aligned}
$$

and, using (A19),

$$
\begin{aligned}
& \beta \mathcal{F}_{\mathrm{ex}}^{(\mathrm{HD})}[\rho] \\
& =\frac{R_{0}}{\pi} \int_{0}^{2 \pi} d \phi\{-\int_{\pi}^{0} d \alpha \Psi(\phi, \alpha)-\underbrace{[\Psi(\phi, \alpha) \theta(\alpha)]_{\pi}^{0}}_{=0}\} \\
& =\frac{R_{0}}{\pi} \int_{0}^{2 \pi} d \phi \int_{0}^{\pi} d \alpha \Psi(\phi, \alpha),
\end{aligned}
$$

where we used the fact that the function $\alpha(\gamma)$ has values $\alpha(0)=\pi$ and $\alpha(\pi)=0$, while the function $\theta(\alpha)$ has values $\theta(0)=\theta(\pi)=0$ (see Fig. 1). Taking into account now that $\frac{\partial \eta_{\mathrm{HD}}}{\partial \alpha}(\phi, \alpha)=R_{0}\left[\rho_{1 \mathrm{D}}(\phi+\alpha)+\rho_{1 \mathrm{D}}(\phi-\alpha)\right]=2 R_{0} n_{0+}(\phi, \alpha)$, we obtain from (A24):

$$
\begin{aligned}
\beta \mathcal{F}_{\mathrm{ex}}^{(\mathrm{HD})}[\rho] & \\
= & -\frac{R_{0}}{\pi} \int_{0}^{2 \pi} d \phi \int_{0}^{\pi} d \alpha \frac{1}{2 R_{0}} \frac{\partial \eta_{\mathrm{HD}}}{\partial \alpha}(\phi, \alpha) \ln \left[1-\eta_{\mathrm{HD}}(\phi, \alpha)\right] \\
= & \frac{1}{2 \pi} \int_{0}^{2 \pi} d \phi \int_{0}^{\pi} d \alpha \frac{\partial}{\partial \alpha}\left\{\eta_{\mathrm{HD}}(\phi, \alpha)\right. \\
& \left.+\left[1-\eta_{\mathrm{HD}}(\phi, \alpha)\right] \ln \left[1-\eta_{\mathrm{HD}}(\phi, \alpha)\right]\right\} \\
= & \frac{1}{2 \pi} \int_{0}^{2 \pi} d \phi\left\{\eta_{\mathrm{HD}}(\phi, \alpha)\right. \\
& \left.+\left[1-\eta_{\mathrm{HD}}(\phi, \alpha)\right] \ln \left[1-\eta_{\mathrm{HD}}(\phi, \alpha)\right]\right\}_{0}^{\pi} .
\end{aligned}
$$

Noting that $\eta_{\mathrm{HD}}(\phi, 0)=0$ and that

$$
\begin{aligned}
\eta_{\mathrm{CA}} & \equiv \eta_{\mathrm{HD}}(\phi, \pi)=R_{0} \int_{\phi-\pi}^{\phi+\pi} d \phi^{\prime} \rho_{1 \mathrm{D}}\left(\phi^{\prime}\right) \\
& =R_{0} \int_{-\pi}^{\pi} d \phi^{\prime} \rho_{1 \mathrm{D}}\left(\phi^{\prime}\right)=2 \pi R_{0} \bar{\rho}_{1 \mathrm{D}},
\end{aligned}
$$

we arrive at (15).

\section{APPENDIX B: FOURIER AND GAUSSIAN PARAMETRIZATIONS}

The expressions for the weighted densities of CRs, using the Fourier expansion (36), are

$$
\begin{aligned}
\eta_{\mathrm{CR}}(\phi, z)= & \eta_{0}\left[1+\sum_{(k, m) \neq(0,0)} s_{k m} \chi_{1}\left(k N_{0} \alpha_{0}\right) \chi_{1}\left(\frac{m \pi}{d^{*}}\right)\right. \\
& \left.\times \cos \left(k N_{0} \phi\right) \cos (q m z)\right], \\
n_{0}^{(\mathrm{CR})}(\phi, z)= & \rho_{0}\left[1+\sum_{(k, m) \neq(0,0)} s_{k m} \chi_{0}\left(k N_{0} \alpha_{0}\right) \chi_{0}\left(\frac{m \pi}{d^{*}}\right)\right. \\
& \left.\times \cos \left(k N_{0} \phi\right) \cos (q m z)\right], \\
n_{1, \perp}^{(\mathrm{CR})}(\phi, z)= & \frac{\eta_{0}}{L}\left[1+\sum_{(k, m) \neq(0,0)} s_{k m} \chi_{1}\left(k N_{0} \alpha_{0}\right) \chi_{0}\left(\frac{m \pi}{d^{*}}\right)\right. \\
& \left.\times \cos \left(k N_{0} \phi\right) \cos (q m z)\right], \\
& \left.\times \cos \left(k N_{0} \phi\right) \cos (q m z)\right], \\
n_{1, \|}^{(\mathrm{CR})}(\phi, z)= & \rho_{0} L\left[1+\sum_{(k, m) \neq(0,0)} s_{k m} \chi_{0}\left(k N_{0} \alpha_{0}\right)\left(\frac{m \pi}{d^{*}}\right)\right.
\end{aligned}
$$

where $\eta_{0}=2 \rho_{0} R_{0} \alpha_{0} L$ is the mean packing fraction, $d^{*}=d / L$ is the $z$ period in reduced units, and $\chi_{0}(x) \equiv \cos x, \chi_{1}(x) \equiv$ $\sin x / x$. 
Within the Gaussian parametrization (37), the free-energy density functional of CR can be computed as

$$
\begin{aligned}
\frac{\beta \mathcal{F}_{\mathrm{CR}}[\rho] a}{2 \pi R_{0} \mathcal{L}}= & \eta_{0}\left\{\ln \left[\frac{\eta_{0} \sqrt{\Lambda_{\perp}^{*} \Lambda_{\|}^{*}}}{\pi a}\right]-1+\frac{2}{\sqrt{\pi}} \int_{0}^{\infty} d t e^{-t^{2}} \ln \left(\prod_{\tau=\perp, \|}\left\{\sum_{k} \exp \left[-\left(t-k \sqrt{\Lambda_{\tau}^{*}}\right)^{2}\right]\right\}\right)\right. \\
& \left.+\frac{1}{\pi} \int_{0}^{\infty} d t_{1} e^{-t_{1}^{2}} \int_{0}^{\infty} d z e^{-t_{2}^{2}} T\left(\frac{t_{1}}{\sqrt{\Lambda_{\perp}} R_{0}}, \frac{t_{2}}{\sqrt{\Lambda_{\|}}}\right)\right\} .
\end{aligned}
$$

where $a=2 R_{0} \alpha_{0} L$ is the particle area and we have defined $\Lambda_{\perp}^{*} \equiv \Lambda\left(\phi_{0}^{*}\right)^{2}\left[\right.$ with $\left.\phi_{0}^{*}=\pi /\left(N_{0} \alpha_{0}\right)\right], \Lambda_{\|}^{*} \equiv \Lambda\left(d^{*}\right)^{2}$, and

$$
T(\phi, z)=\sum_{\tau_{1}, \tau_{2}= \pm} H\left(\phi_{\tau_{1}}, z_{\tau_{2}}\right), \quad H(\phi, z)=-\ln \left[1-\eta_{\mathrm{CR}}(\phi, z)\right]+\frac{\eta_{\mathrm{CR}}(\phi, z)}{1-\eta_{\mathrm{CR}}(\phi, z)},
$$

with $\phi_{ \pm}=\phi \pm \alpha_{0}, z=z \pm L / 2$, and $\eta_{\mathrm{CR}}(\phi, z)=(1-v) \zeta_{1 \perp}(\phi) \zeta_{1 \|}(z)$. We have used the notation

$$
\begin{aligned}
& \zeta_{1 \perp}\left(\frac{t_{1}}{\sqrt{\Lambda_{\perp}} R_{0}}\right)=\frac{1}{2} \sum_{k}\left\{\operatorname{erf}\left[t_{1}+\sqrt{\Lambda_{\perp}^{*}}\left(\frac{1}{2 \phi_{0}^{*}}-k\right)\right]-\operatorname{erf}\left[t_{1}-\sqrt{\Lambda_{\perp}^{*}}\left(\frac{1}{2 \phi_{0}^{*}}+k\right)\right]\right\}, \\
& \zeta_{1 \|}\left(\frac{t_{2}}{\sqrt{\Lambda_{\|}}}\right)=\frac{1}{2} \sum_{k}\left\{\operatorname{erf}\left[t_{2}+\sqrt{\Lambda_{\|}^{*}}\left(\frac{1}{2 d^{*}}-k\right)\right]-\operatorname{erf}\left[t_{2}-\sqrt{\Lambda_{\|}^{*}}\left(\frac{1}{2 d^{*}}+k\right)\right]\right\},
\end{aligned}
$$

with $\operatorname{erf}(x)$ the error function.

\section{APPENDIX C: DF FOR SPHERICAL LENSES}

The correct dimensional crossover of HSs confined on a spherical surface means the following: When the density profile of HSs is restricted to be on a spherical surface of radius $R_{0}, \rho_{3 \mathrm{D}}(r, \hat{\boldsymbol{\Omega}})=\rho_{2 \mathrm{D}}(\hat{\boldsymbol{\Omega}}) \delta\left(R_{0}-r\right)$, where $(r, \theta, \phi)$ are the radius and the angles of spherical coordinates, while $\hat{\mathbf{\Omega}}(\theta, \phi)=(\sin \theta \cos \phi, \sin \theta \sin \phi, \cos \theta)$ is the unit vector in the radial direction, we should obtain

$$
\beta \mathcal{F}_{\mathrm{ex}}^{(\mathrm{HS})}[\rho] \rightarrow \beta \mathcal{F}_{\mathrm{ex}}^{(\mathrm{SL})}[\rho]=R_{0}^{2} \int d \hat{\mathbf{\Omega}} \Phi_{2 \mathrm{D}}^{(\mathrm{SL})}(\hat{\mathbf{\Omega}}),
$$

which results in a dimensionally reduced density functional for spherical lenses (SLs), where $d \hat{\mathbf{\Omega}}=d \phi d \theta \sin \theta$ is the solid-angle element. The expression for $\Phi_{2 \mathrm{D}}(\hat{\boldsymbol{\Omega}})$ is the same as in (2), with the weighted densities now defined as

$$
\begin{gathered}
n_{0}^{(\mathrm{SL})}(\hat{\boldsymbol{\Omega}})=\frac{R_{0}}{2 \pi R} \int d \hat{\boldsymbol{\Omega}}_{1} \rho_{2 \mathrm{D}}\left(\hat{\boldsymbol{\Omega}}_{1}\right) \delta\left(\alpha_{0}-\gamma\left(\hat{\boldsymbol{\Omega}}, \hat{\boldsymbol{\Omega}}_{1}\right)\right), \\
\eta_{\mathrm{SL}}(\hat{\boldsymbol{\Omega}})=R_{0}^{2} \int d \hat{\boldsymbol{\Omega}}_{1} \rho_{2 \mathrm{D}}\left(\hat{\boldsymbol{\Omega}}_{1}\right) \Theta\left(\alpha_{0}-\gamma\left(\hat{\boldsymbol{\Omega}}, \hat{\boldsymbol{\Omega}}_{1}\right)\right), \\
N_{\mathrm{SL}}(\hat{\boldsymbol{\Omega}})=\left(\frac{R_{0}}{2 \pi R}\right)^{2} \int d \hat{\boldsymbol{\Omega}}_{1} \int d \hat{\boldsymbol{\Omega}}_{2} \rho_{2 \mathrm{D}}\left(\hat{\boldsymbol{\Omega}}_{1}\right) \rho_{2 \mathrm{D}}\left(\hat{\boldsymbol{\Omega}}_{2}\right) \delta\left(\alpha_{0}-\gamma\left(\hat{\boldsymbol{\Omega}}, \hat{\boldsymbol{\Omega}}_{1}\right)\right) \delta\left(\alpha_{0}-\gamma\left(\hat{\boldsymbol{\Omega}}, \hat{\boldsymbol{\Omega}}_{2}\right)\right) \mathcal{K}\left(\frac{R \gamma\left(\hat{\boldsymbol{\Omega}}_{1}, \hat{\boldsymbol{\Omega}}_{2}\right)}{\alpha_{0}}\right),
\end{gathered}
$$

where $\alpha_{0}=\sin ^{-1}\left(R / R_{0}\right)$ and we have used the notations

$$
\begin{gathered}
\cos \gamma\left(\hat{\mathbf{\Omega}}, \hat{\boldsymbol{\Omega}}_{i}\right)=\sin \theta \sin \theta_{i} \cos \left(\phi-\phi_{i}\right)+\cos \theta \cos \theta_{i}, \quad i=1,2, \\
\cos \gamma\left(\hat{\boldsymbol{\Omega}}_{1}, \hat{\mathbf{\Omega}}_{2}\right)=\sin \theta_{1} \sin \theta_{2} \cos \left(\phi_{1}-\phi_{2}\right)+\cos \theta_{1} \cos \theta_{2},
\end{gathered}
$$

which define the angles between the unit vectors $\hat{\mathbf{\Omega}}$ and $\hat{\boldsymbol{\Omega}}_{i}$ and $\hat{\boldsymbol{\Omega}}_{1}$ and $\hat{\mathbf{\Omega}}_{2}$, respectively. The kernel $\mathcal{K}(r)$ is that defined in Eq. (6). The conditions $\rho(\phi, \theta)=\rho(\phi+2 \pi, \theta), \rho(\phi, \theta)=\rho(\phi, \theta+2 \pi)$ should be imposed, as the density profile is now a periodic function of $\phi$ and $\theta$.

[1] D. R. Nelson, Defects and Geometry in Condensed Matter Physics (Cambridge University Press, Cambridge, UK, 2002).

[2] D. R. Nelson, Phys. Rev. B 28, 5515 (1983).

[3] M. J. Bowick, D. R. Nelson, and A. Travesset, Phys. Rev. B 62, 8738 (2000).
[4] A. R. Bausch, M. J. Bowick, A. Cacciuto, A. D. Dinsmore, M. F. Hsu, D. R. Nelson, M. G. Nikolaides, A. Travesset, and D. A. Weitz, Science 299, 1716 (2003).

[5] J. Lidmar, L. Mirny, and D. R. Nelson, Phys. Rev. E 68, 051910 (2003). 
[6] S. Sachdev and D. R. Nelson, J. Phys. C 17, 5473 (1984).

[7] A. D. Dinsmore, D. T. Wong, P. Nelson, and A. G. Yodh, Phys. Rev. Lett. 80, 409 (1998).

[8] A. Amir, J. Paulose, and D. R. Nelson, Phys. Rev. E 87, 042314 (2013).

[9] J. Dzubiella, M. Schmidt, and H. Löwen, Phys. Rev. E 62, 5081 (2000).

[10] A. González, J. A. White, F. L. Román, and S. Velasco, J. Chem. Phys. 125, 064703 (2006).

[11] D. de las Heras, E. Velasco, and L. Mederos, Phys. Rev. Lett. 94, 017801 (2005).

[12] D. de las Heras, E. Velasco, and L. Mederos, Phys. Rev. E 74, 011709 (2006).

[13] A. Mughal, H. K. Chan, and D. Weaire, Phys. Rev. Lett. 106, 115704 (2011).

[14] T. Franosch, S. Lang, and R. Schilling, Phys. Rev. Lett. 109, 240601 (2012).
[15] Y. Rosenfeld, M. Schmidt, H. Lowen, and P. Tarazona, Phys. Rev. E 55, 4245 (1997).

[16] P. Tarazona and Y. Rosenfeld, Phys. Rev. E 55, R4873 (1997).

[17] J. A. Cuesta and Y. Martínez-Ratón, Phys. Rev. Lett. 78, 3681 (1997).

[18] Y. Martínez-Ratón, J. A. Capitán, and J. A. Cuesta, Phys. Rev. E 77, 051205 (2008).

[19] A. V. Kityk and P. Huber, Appl. Phys. Lett. 97, 153124 (2010).

[20] S. Belli, M. Dijkstra, and R. van Roij, J. Chem. Phys. 137, 124506 (2012).

[21] W. G. Hoover, C. G. Hoover, and M. N. Bannermann, J. Stat. Phys. 136, 715 (2009).

[22] J. A. Capitán, Y. Martínez-Ratón, and J. A. Cuesta, J. Chem. Phys. 128, 194901 (2008).

[23] A. Amir and D. Nelson, Proc. Natl. Acad. Sci. USA 109, 9833 (2012). 